\title{
ON THE RATE OF CONVERGENCE OF MOMENTS IN THE CENTRAL LIMIT THEOREM
}

\author{
PETER HALL \\ (Received 10 October 1977) \\ Communicated by J. Gani
}

\begin{abstract}
An early extension of Lindeberg's central limit theorem was Bernstein's (1939) discovery of necessary and sufficient conditions for the convergence of moments in the central limit theorem. Von Bahr (1965) made a study of some asymptotic expansions in the central limit theorem, and obtained rates of convergence for moments. However, his results do not in general imply that the moments converge. Some better rates have been obtained by Bhattacharya and Rao for moments between the second and third. In this paper we give improved rates of convergence for absolute moments between the third and fourth.
\end{abstract}

Subject classification (Amer. Math. Soc. (MOS) 1970): primary $60 \mathrm{~F} 05$; secondary $60 \mathrm{G} 50$.

\section{Introduction and summary}

The moments of a distribution are among its most useful and accessible characteristics. Hence it is not surprising that one of the earliest extensions of Lindeberg's central limit theorem was Bernstein's (1939) discovery of a necessary and sufficient condition for the convergence of absolute moments in the central limit theorem. Bernstein's theorem may be stated as follows: Suppose that for each $n \geqslant 1, X_{n 1}, X_{n 2}, \ldots, X_{n k_{n}}$ are independent random variables with zero means and finite variances satisfying $\sum_{j} E X_{n j}^{2}=1$, and that the $X_{n j}$ are asymptotically negligible in the sense that $\max _{j \leqslant k_{n}} P\left(\left|X_{n j}\right|>\varepsilon\right) \rightarrow 0$ as $n \rightarrow \infty$ for all $\varepsilon>0$. Then

$$
S_{n}=\sum_{j} X_{n j} \stackrel{\mathscr{Q}}{\longrightarrow} N(0,1)
$$

and the $p$ th absolute moment of $S_{n}(p>2)$ converges to that of the standard normal distribution if and only if

$$
L_{n}=\sum_{j} E\left|X_{n j}\right|^{p \rightarrow 0}
$$

Brown $(1969,1970)$ gave a characteristic function proof of Bernstein's result. Von Bahr (1965) made a study of some asymptotic expansions in the central limit 
theorem and their application to the rate of convergence of moments. Bhattacharya and Rao (1976) have improved on some of von Bahr's results in the case $2<p \leqslant 3$. In this connection see also Michel (1976), who tackles the problem via non-uniform estimates of the rate of convergence in the central limit theorem. Our aim in this paper is to obtain simplified rates of convergence of moments, especially in the case $3<p \leqslant 4$.

Let $\mu_{p}(p>2)$ denote the $p$ th absolute moment of a standard normal variable. If $p$ is an even integer it is well known that the rate of convergence of $E\left|S_{n}\right|^{p}$ to $\mu_{p}$ depends on whether earlier moments match those of the standard normal distribution. For example, if $S_{n}=\sum_{1}^{n} X_{j} / \sqrt{n}$ where the $X_{j}$ are i.i.d. (independent and identically distributed) with finite sixth moments and with third and fourth moments equal to $m_{3}$ and $m_{4}$ respectively, then

$$
E S_{n}^{6}=15+\left\{10 m_{3}^{2}+15\left(m_{4}-3\right)\right\} / n+O\left(n^{-2}\right) .
$$

If $m_{4}=\mu_{4}=3$ and $m_{3}=0$ then the rate of convergence is $O\left(n^{-2}\right)$; if $10 m_{3}^{2}+15\left(m_{4}-3\right) \neq 0$ then the rate is $O\left(n^{-1}\right)$. When $p>3$ and the $X_{j}$ are not i.i.d., it is more difficult to see how to frame the results so as to obtain a good rate of convergence of $E\left|S_{n}\right|^{p}$ to $\mu_{p}$. We will confine our attention to the range $2<p \leqslant 4$. Our results are stated in Section 2 and the proofs given in Section 3.

\section{Rates of convergence}

Von Bahr's most general rates of convergence are given in his Theorem 4, and in the case $2<p \leqslant 4$ they can be summarized as follows: If $k_{n}=n$ then for all $q \leqslant p$,

$$
\left.|E| S_{n}\right|^{q}-\mu_{q} \mid= \begin{cases}O\left(n^{(p-2) / 2} L_{n}^{2}+L_{n}^{(q+1) /(p-2)}\right), & \text { if } 2<p<3, \\ O\left(n^{(p-2) / 2} L_{n}^{2}+n^{(q+1)(p-3) / p} L_{n}^{3(q+1) / p}\right), & \text { if } 3 \leqslant p \leqslant 4 .\end{cases}
$$

(Actually von Bahr's results apply in the case when $X_{n j}=X_{j}\left(\sum_{1}^{n} E X_{j}^{2}\right)^{-t}$, where $\left\{X_{n}, n \geqslant 1\right\}$ is a sequence of independent random variables. Also, his result in the case $2<p<3$ is rather more complicated than that given here.) In general this does not imply that $E\left|S_{n}\right|^{q} \rightarrow \mu_{q}$, although Bernstein's work tells us that this $i$ the case for all $q \leqslant p$, provided only that $L_{n} \rightarrow 0$ and that the random variables in each row are asymptotically negligible. In the cases (a) $0<q \leqslant p=3$, and (b) $0<q \leqslant 2$, $2<p \leqslant 3$, Bhattacharya and Rao (1976), Theorem 18.1, have shown that $\left.|E| S_{n}\right|^{q}-\mu_{q} \mid \leqslant C d_{n, p} \leqslant C L_{n}$, where the constant $C$ depends only on $p$ and $q$, and

$$
d_{n, p}=\inf _{0 \leqslant 8 \leqslant 1}\left[\varepsilon^{3-p} \sum_{j} \int_{\left\{\left|X_{n j}\right| \leqslant \varepsilon\right\}}\left|X_{n j}\right|^{p} d P+\sum_{j} \int_{\left\{\mid X_{n j} \backslash>8\right\}}\left|X_{n j}\right|^{p} d P\right] \text {. }
$$

In case (b) when the $X_{j}$ are i.i.d. this yields an error bound of $o\left(n^{-(p-2) / 2}\right)$, slightly improving on the $O\left(n^{-(p-2) / 2}\right)$ bound due to von Bahr. However, there is no such improvement in case (a). 
Our results are most significant in the case $3<p<4$, where there is no published improvement of von Bahr's. We state them for the larger range $2<p \leqslant 4$. They contain those of von Bahr in the i.i.d. case, except when $0<q<p-2$, when his rates are slightly better than ours. The reason for this is that we have used a simpler method for estimating the integrals of the tails of characteristic functions. (See his remark on p. 817.) In the case of a general triangular array this meant a considerable simplification of the proofs and the results.

THEOREM. Let $X_{n j}, 1 \leqslant j \leqslant k_{n}$, be a sequence of independent random variables with zero means and finite variances satisfying $\sum_{j} E\left(X_{n j}^{2}\right)=1$, and let $S_{n}=\Sigma_{j} X_{n j}$. Suppose that $L_{n}=\Sigma_{j} E\left|X_{n j}\right|^{p}<\infty$, where $2<p<4$. Let $\mu_{p}$ denote the absolute pth moment of the standard normal distribution. There exists an absolute constant $C_{0}$ such that for all $q$ in $0<q \leqslant p$, and whenever $L_{n} \leqslant 1$ (and $\left|E S_{n}^{3}\right| \leqslant 1$ if $p>3$ ),

$$
\left.|E| S_{n}\right|^{q}-\mu_{q}\left|\leqslant C_{0}^{1 /(p-2)}\right| \sin \left(\frac{1}{2} q \pi\right) \mid M_{n} / q(4-p),
$$

where

$$
M_{n}= \begin{cases}L_{n}+L_{n}^{q /(p-2)}, & \text { if } 2<p \leqslant 3, \\ L_{n}+L_{n}^{q /(p-2)}+\left|E S_{n}^{3}\right|^{2}, & \text { if } 3<p<4 .\end{cases}
$$

In the case $p=4,\left.|E| S_{n}\right|^{4}-\mu_{4} \mid \leqslant C_{0}^{\ddagger}\left(\sum_{j} E X_{n j}^{4}+\left|E S_{n}^{3}\right|^{2}\right)$ if $\sum_{j} E X_{n j}^{4} \leqslant 1$ and $\left|E S_{n}^{\mathbf{3}}\right| \leqslant 1$.

REMARK. It follows from von Bahr's Theorem 2 that in the i.i.d. case, $\left.|E| S_{n}\right|^{3}-\mu_{3} \mid=O\left(n^{-1}\right)$ when $X_{1}$ has finite fourth moment, whereas $\left|E S_{n}^{3}\right|=O\left(n^{-1}\right)$ if $E\left(X_{1}^{3}\right) \neq 0$. Hence the rate of convergence of $E\left|S_{n}\right|^{p}$ to $\mu_{p}$ may be considerably faster than the rate of convergence of $\left|E S_{n}^{3}\right|$ to 0 , although in general it will not be faster than the rate of convergence of $\left|E S_{n}^{3}\right|^{2}$ to 0 .

\section{The proofs}

We will begin by establishing two lemmas. In our proofs the symbol $C$ denotes a generic constant depending only on $p$, not necessarily the same at each appearance, while $C_{1}, C_{2}, C_{3}$ and $C_{4}$ denote particular versions of $C$. The symbols $\theta, \varphi$ and $\psi$ denote generic complex- or real-valued functions of the real variable $t$, dominated by 1 for all $t . \mathrm{Rl} z$ denotes the real part of $z$.

Let $f_{n j}$ and $f_{n}$ be the characteristic functions of $X_{n j}$ and $S_{n}$, respectively, and let $r_{n j}=f_{n j}-1$. If $E\left|S_{n}\right|^{p}<\infty$ we will write

$$
\begin{aligned}
f_{n j}(t) & =1+\sum_{r=2}^{m}(i t)^{r} E X_{n j}^{r} / r !+\alpha_{n j}(t), \\
f_{n}(t) & =1+\sum_{r=2}^{m}(i t)^{r} E S_{n}^{r} / r !+\alpha_{n}(t)
\end{aligned}
$$

and

$$
e^{-1 t^{2}}=1-\frac{1}{2} t^{2}+\alpha(t)
$$


where $m=2$ if $2<p \leqslant 3, m=3$ if $3<p \leqslant 4$, and $\alpha_{n j}(t)$ is of the form

$$
\alpha_{n j}(t)=C \theta_{n j}(t)|t|^{p} E\left|X_{n j}\right|^{p} \text {. }
$$

(See Loève (1960), p. 199.)

LeмmA 1. There exist constants $A_{p}$ and $B_{p}$ depending only on $p$ such that whenever $2<p<4,0 \leqslant q \leqslant p, L_{n} \leqslant 1$ (and $\left|E S_{n}^{3}\right| \leqslant 1$ if $p>3$ ),

$$
\left|\int_{0}^{B_{j}}\left(\mathrm{R} 1 f_{n}(t)-e^{-1 i^{2}}\right) t^{-(\alpha+1)} d t\right| \leqslant A_{p} M_{n} /(4-p) .
$$

$A_{p}$ is bounded away from $\infty$ and $B_{p}$ is bounded away from zero.

Proof. We will give a proof in the case $3<p<4$. Now,

$$
\begin{aligned}
\left|r_{n j}\right| & \leqslant \frac{1}{2} t^{2} E\left(X_{n j}^{2}\right)+\frac{1}{6}|t|^{3} E\left|X_{n j}\right|^{3}+C|t|^{p} E\left|X_{n j}\right|^{p} \\
& \leqslant C_{1}\left\{t^{2}\left(E\left|X_{n j}\right|^{p}\right)^{2 / p}+|t|^{3}\left(E\left|X_{n j}\right|^{p}\right)^{3 / p}+|t|^{p} E\left|X_{n j}\right|^{p}\right\} \\
& \leqslant C_{1}\left\{t^{2} L_{n}^{2 / p}+|t|^{3} L_{n}^{3 / p}+|t|^{p} L_{n}\right\} \leqslant \frac{1}{2}
\end{aligned}
$$

if $|t| \leqslant B_{p}^{(1)} L_{n}^{-1 / p}$, where $B_{p}^{(1)}=\min \left\{1,\left(6 C_{1}\right)^{-1}\right\}$. Furthermore,

$$
\begin{aligned}
\left|r_{n j}\right|^{2} & \leqslant 4\left\{\left(\frac{1}{2} t^{2} E X_{n j}^{2}\right)^{2}+\left(\frac{1}{6}|t|^{3} E\left|X_{n j}\right|^{3}\right)^{2}+\left(C|t|^{p} E\left|X_{n j}\right|^{p}\right)^{2}\right\} \\
& \leqslant C|t|^{4}\left\{\left(E\left|X_{n j}\right|^{p}\right)^{4 / p}+\left(E\left|X_{n j}\right|^{p}\right)^{6 / p}+\left(E\left|X_{n j}\right|^{p}\right)^{2}\right\} \quad \text { if } t \leqslant 1 \\
& \leqslant C|t|^{4} E\left|X_{n j}\right|^{p}
\end{aligned}
$$

if $p \leqslant 4$ and $L_{n} \leqslant 1$. It follows that if $|t| \leqslant B_{p}^{(1)}$ and $L_{n} \leqslant 1$ then the principal-valued logarithm of $f_{n j}$ is given by

$$
\log f_{n j}(t)=\frac{1}{2}(i t)^{2} E X_{n j}^{2}+\frac{1}{6}(i t)^{3} E X_{n j}^{3}+\alpha_{n j}(t)+C \theta_{n j}(t)|t|^{4} E\left|X_{n j}\right|^{p}
$$

and that of $f_{n}$ is given by

$$
\log f_{n}(t)=-\frac{1}{2} t^{2}-\frac{1}{6} i t^{3} E S_{n}^{3}+\sum_{j} \alpha_{n j}(t)+C \theta_{n}(t)|t|^{4} L_{n}
$$

Hence if $|t| \leqslant B_{p}^{(1)}$ and $L_{n} \leqslant 1$,

say. Now,

$$
\begin{aligned}
f_{n}(t) & =e^{-t i^{2}} \exp \left\{-\frac{1}{6} i t^{3} E S_{n}^{3}+\sum_{j} \alpha_{n j}(t)+C \theta_{n}(t)|t|^{4} L_{n}\right\} \\
& =e^{-\frac{1}{3} r^{2}} \exp \left(s_{n}\right),
\end{aligned}
$$

$$
\left|s_{n}\right| \leqslant C_{2}\left\{|t|^{3}\left|E S_{n}^{3}\right|+|t|^{p} L_{n}+|t|^{4} L_{n}\right\} \leqslant \frac{1}{2}
$$

if $L_{n} \leqslant 1,\left|E S_{n}^{3}\right| \leqslant 1$ and $|t| \leqslant B_{p}=\min \left\{B_{p}^{(1)},\left(6 C_{2}\right)^{-1}\right\}$. Also,

$$
\left|s_{n}\right|^{2} \leqslant C|t|^{4}\left(L_{n}+\left|E S_{n}^{3}\right|^{2}\right)
$$

if $L_{n} \leqslant 1$ and $|t| \leqslant 1$, and so

$$
f_{n}(t)=e^{-t^{2}}\left\{1-\frac{1}{6} i t^{3} E S_{n}^{3}+\sum_{j} \alpha_{n j}(t)+C \varphi_{n}(t)|t|^{4}\left(L_{n}+\left|E S_{n}^{3}\right|^{2}\right)\right\}
$$


Consequently,

$$
\mathrm{Rl} f_{n}(t)-e^{-\mathrm{d} t^{2}}=e^{-\left.\mathrm{b}\right|^{2}} \sum_{j} \mathrm{Rl} \alpha_{n j}(t)+C \psi_{n}(t)|t|^{4}\left(L_{n}+\left|E S_{n}^{3}\right|^{2}\right) .
$$

The functions $\mathrm{Rl} \alpha_{n j}(t)=E\left\{\cos \left(t X_{n j}\right)-1+\frac{1}{2} t^{2} X_{n j}^{2}\right\}$ are non-negative, and since $B_{p} \leqslant 1$,

$$
\begin{aligned}
& ||_{0}^{B_{p}}\left(\mathrm{R} 1 f_{n}(t)-e^{-t^{2}}\right) t^{-(q+1)} d t \mid \\
& \quad \leqslant\left.\sum_{j}\right|_{0} ^{B_{p}} \mathrm{Rl} \alpha_{n j}(t) t^{-(p+1)} d t+\left.C\left(L_{n}+\left|E S_{n}^{3}\right|^{2}\right)\right|_{0} ^{B_{p}} t^{3-p} d t .
\end{aligned}
$$

For each $\lambda>0$,

$$
\int_{0}^{\lambda} \mathrm{Rl} \alpha_{n j}(t) t^{-(p+1)} d t=E\left(\left|X_{n j}\right|^{p} A\left(\lambda\left|X_{n j}\right|\right)\right],
$$

where $A(x)=\int_{0}^{x}\left(\cos t-1+\frac{1}{2} t^{2}\right) t^{-(p+1)} d t$ is a continuous bounded increasing function with $A(0)=0$ and $A(\infty)=\pi / 2 \Gamma(p+1)\left|\sin \frac{1}{2} p \pi\right|$. See Pitman (1968), p. 428.) Hence

$$
\begin{gathered}
||_{0}^{B_{p}}\left(\operatorname{R} 1 f_{n}(t)-e^{-1 k^{2}}\right) t^{-(q+1)} d t \mid \\
\leqslant C\left\{\left(L_{n} /\left|\sin \frac{1}{2} p \pi\right|\right)+B_{p}^{4-q}\left(L_{n}+\left|E S_{n}^{3}\right|^{2}\right) /(4-p)\right\} \\
\leqslant A_{p}\left(L_{n}+\left|E S_{n}^{3}\right|^{2}\right) /(4-p),
\end{gathered}
$$

where $A_{p}$ is bounded as $p \rightarrow 4$. $B_{p}$ is bounded away from zero. This completes the proof of Lemma 1.

Lemma 2. There exist constants $A_{p}$ and $B_{p}$ depending only on $p$ such that whenever $2<p \leqslant 4$ and $|t| \leqslant B_{p} L_{n}^{-1 /(p-2)}$,

$$
\left|\mathrm{R} 1 f_{n}(t)-e^{-1 / t^{2}}\right| \leqslant A_{p}\left(t^{6}+|t|^{p}\right) e^{-i^{2} / 3} M_{n} .
$$

$A_{p}$ is bounded away from $\infty$ and $B_{p}^{p-2}$ is bounded away from zero.

Proof. We will give a proof in the case $3<p \leqslant 4$. As in the proof of Lemma 1 , we can show that if $|t| \leqslant C_{1} L_{n}^{-1 / p}$ then $\left|r_{n j}\right| \leqslant \frac{1}{2}$. Furthermore,

$$
\left|r_{n j}\right|^{2} \leqslant 4\left\{\left(|t|^{p} E\left|X_{n j}\right|^{p}\right)^{4 / p}+\left(|t|^{p} E\left|X_{n j}\right|^{p}\right)^{6 / p}+\left(|t|^{p} E\left|X_{n j}\right|^{p}\right)^{2}\right\},
$$

and so if $|t| \leqslant C_{1} L_{p}^{-1 / p} \leqslant C_{1}\left(E\left|X_{n j}\right|^{p}\right)^{-1 / p}$ then

$$
\left|r_{n j}\right|^{2} \leqslant 4\left\{C_{1}^{4-p}+C_{1}^{6-p}+C_{1}^{p}\right\}|t|^{p} E\left|X_{n j}\right|^{p} .
$$

Consequently the principal-valued logarithm of $f_{n j}$ is given by

$$
\log f_{n j}(t)=\frac{1}{2}(i t)^{2} E X_{n j}^{2}+\frac{1}{6}(i t)^{3} E X_{n j}^{3}+C \theta_{n j}(t)|t|^{p} E\left|X_{n j}\right|^{p}
$$

and that of $f_{n}$, by

$$
\log f_{n}(t)=-\frac{1}{2} t^{2}-\frac{1}{8} i t^{3} E S_{n}^{3}+C_{2} \theta_{n}(t)|t|^{p} L_{n}
$$


Hence if $|t| \leqslant C_{1} L_{n}^{-1 / p}$ then

$$
\begin{aligned}
\left|\mathrm{R} 1 f_{n}(t)-e^{-\frac{1}{2} t^{2}}\right| & \leqslant\left|f_{n}(t)-\exp \left(-\frac{1}{2} t^{2}-\frac{1}{6} i t^{3} E S_{n}^{3}\right)\right| \\
& +\mid \mathrm{R} 1 \exp \left(-\frac{1}{2} t^{2}-\frac{1}{6} i t^{3} E S_{n}^{3}\right)-e^{-\frac{1}{2} t^{2} \mid} \\
& \leqslant C_{2}|t|^{p} L_{n} \exp \left(-\frac{1}{2} t^{2}+C_{2}|t|^{p} L_{n}\right)+e^{-1 / 2}\left\{1-\cos \left(\frac{1}{6} t^{3} E S_{n}^{3}\right)\right\} \\
& \leqslant C_{2}|t|^{p} e^{-t^{2} / 3} L_{n}+t^{6} e^{-\frac{1}{2} t^{2}}\left|E S_{n}^{3}\right|^{2}
\end{aligned}
$$

if $|t| \leqslant\left(6 C_{2} L_{n}\right)^{-1 /(p-2)}$. Let $A_{p}^{(1)}=C_{2}+1$ and $B_{p}^{(1)}=\left(6 C_{2}\right)^{-1 /(p-2)}$. If

then

$$
|t| \leqslant \min \left\{C_{1} L_{n}^{-1 / p}, B_{p}^{(1)} L_{n}^{-1 /(p-2)}\right\}
$$

$$
\left|\mathrm{Rl} f_{n}(t)-e^{-\frac{1}{2} t^{2}}\right| \leqslant A_{p}^{(1)}\left(t^{6}+|t|^{p}\right) \mathrm{e}^{-t^{2} / 3} M_{n} \text {. }
$$

Now suppose that $|t|>C_{1} L_{n}^{-1 / p}$. Let $\tilde{X}_{n j}=X_{n j}-Y_{n j}$, where $Y_{n j}$ is independent of $X_{n j}$ but with the same distribution. Then

so that

$$
E \tilde{X}_{n j}^{2}=2 E X_{n j}^{2}, \quad E \tilde{X}_{n j}^{3}=0 \text { and } E\left|\tilde{X}_{n j}\right|^{p} \leqslant 2^{p} E\left|X_{n j}\right|^{p} \text {, }
$$

$$
\begin{aligned}
\left|f_{n j}(t)\right|^{2} & =1-\frac{1}{2} t^{2} E \tilde{X}_{n j}^{2}+C \theta_{n j}|t|^{p} E\left|\tilde{X}_{n j}\right|^{p} \\
& \leqslant 1-t^{2} E X_{n j}^{2}+C_{3}|t|^{p} E\left|X_{n j}\right|^{p} \\
& \leqslant \exp \left(-t^{2} E X_{n j}^{2}+C_{3}|t|^{p} E\left|X_{n j}\right|^{p}\right) .
\end{aligned}
$$

Consequently

$$
\left|f_{n}(t)\right|^{2}=\prod_{j}\left|f_{n j}(t)\right|^{2} \leqslant \exp \left(-t^{2}+C_{3}|t|^{p} L_{n}\right) \leqslant e^{-2 e^{2} / 3}
$$

if $|t| \leqslant\left(3 C_{3} L_{n}\right)^{-1 /(p-2)}$. Let $B_{p}^{(2)}=\left(3 C_{3}\right)^{-1 /(p-2)}$. If

then

$$
C_{1} L_{n}^{-1 / p}<|t| \leqslant B_{p}^{(2)} L_{n}^{-1 /(p-2)}
$$

$$
\begin{aligned}
\left|f_{n}(t)-e^{-\frac{1}{2} t^{2}}\right| & \leqslant\left|f_{n}(t)\right|+e^{-\frac{1}{2} t^{2}} \\
& \leqslant 2 e^{-t^{2} / 3} \\
& \leqslant 2 C_{1}^{-p}|t|^{p} e^{-t^{2} / 3} L_{n}
\end{aligned}
$$

Let $A_{p}^{(2)}=2 C_{1}^{-p}, A_{p}=\max \left\{A_{p}^{(1)}, A_{p}^{(2)}\right\}$ and $B_{p}=\min \left\{B_{p}^{(1)}, B_{p}^{(2)}\right\}$ to obtain the desired result.

Proof OF THEOREM. Suppose that $L_{n} \leqslant 1,\left|E S_{n}^{3}\right| \leqslant 1,0<q \leqslant p<4$ and $q \neq 2$. Let $k_{q}=\pi /\left[\Gamma(q+1)\left|\sin \frac{1}{2} q \pi\right|\right]$. By Lemma 1 of Brown (1970),

$$
\begin{aligned}
\left.k_{q}|E| S_{n}\right|^{q}-\mu_{q} \mid & =\left|\int_{0}^{\infty}\left(\mathrm{R} l \alpha_{n}(t)-\alpha(t)\right) t^{-(q+1)} d t\right| \\
& =\left|\int_{0}^{\infty}\left(\mathrm{R} l f_{n}(t)-e^{-\frac{1}{2} t^{2}}\right) t^{-(q+1)} d t\right| \\
& \leqslant\left|\int_{0}^{C_{1}}\left(\mathrm{R} 1 f_{n}(t)-e^{-\frac{1}{2} q^{2}}\right) t^{-(q+1)} d t\right| \\
& \quad+\int_{C_{1}}^{C_{2}}\left|\mathrm{R} 1 f_{n}(t)-e^{-\frac{1}{2} l^{2}}\right| t^{-(q+1)} d t+2 \int_{C_{2}}^{\infty} t^{-(q+1)} d t
\end{aligned}
$$


for any constants $C_{1} \leqslant C_{2}$. Our Lemmas 1 and 2 now imply that

$$
\begin{aligned}
& \left.k_{q}|E| S_{n}\right|^{q}-\mu_{q} \mid \\
& \quad \leqslant C_{3}\left\{\left(M_{n} /(4-p)\right)+M_{n} \int_{C_{1}}^{\infty}\left(t^{6}+|t|^{p}\right) e^{-\varepsilon^{2} / 3} d t+\int_{C_{4} L_{n}-1 /(p-2)}^{\infty} t^{-(q+1)} d t\right\},
\end{aligned}
$$

where $C_{3}$ is bounded away from $\infty$, and $C_{1}$ and $C_{4}^{p-2}$ are bounded away from zero,

$$
\leqslant C^{1 /(p-2)} M_{n} / q(4-p),
$$

where $C$ is bounded away from $\infty$. Hence

$$
\left.|E| S_{n}\right|^{q}-\mu_{q}\left|\leqslant C_{0}^{1 /(p-2)}\right| \sin \frac{1}{2} q \pi \mid M_{n} / q(4-p),
$$

where $C_{0}$ is an absolute constant. By setting $q=p$ and taking the limit as $p \uparrow 4$, and noting that $E|X|^{p} \rightarrow E|X|^{4}$ as $p \uparrow 4$, we see that

$$
\begin{aligned}
\left.|E| S_{n}\right|^{4}-\mu_{4} \mid & \leqslant C_{0}^{\ddagger}\left(\frac{1}{2} \pi\right)\left(2 L_{n}+\left|E S_{n}^{3}\right|^{2}\right) / 4 \\
& \leqslant C_{0}^{\ddagger}\left(L_{n}+\left|E S_{n}^{3}\right|^{2}\right),
\end{aligned}
$$

provided that $L_{n}=\Sigma_{j} E X_{n j}^{4} \leqslant 1$ and $\left|E S_{n}^{3}\right| \leqslant 1$.

\section{REFERENCES}

B. von Bahr (1965), "On the convergence of moments in the central limit theorem", Ann. Math. Statist. 36, 808-818.

S. Bernstein (1939), "Quelques remarques sur le théorème limite Liapounoff', Dokl. Akad. Nauk SSR (Comptes rendus) 24, 3-8.

R. N. Bhattacharya and R. R. Rao (1976), Normal Approximation and Asymptotic Expansions (Wiley, New York).

B. M. Brown (1969), "Moments of a stopping rule related to the central limit theorem", Ann. Math. Statist. 40, 1236-1249.

B. M. Brown (1970), "Characteristic functions, moments and the central limit theorem", Ann. Math. Statist. 41, 658-664.

M. Loève (1960), Probability Theory (Van Nostrand, Princeton).

R. Michel (1976), "Nonuniform central limit bounds with applications to probabilities of deviations", Ann. Probability 4, 102-106.

E. J. G. Pitman (1968), "On the behaviour of the characteristic function of a probability distribution in the neighbourhood of the origin", J. Austral. Math. Soc. 8, 423-443.

Department of Statistics

University of Melbourne

Parkville, Victoria 3052

Australia 